\title{
RP-HPLC METHOD DEVELOPMENT, VALIDATION, AND QUANTIFICATION OF LORNOXICAM IN LIPID NANOPARTICLE FORMULATIONS
}

\author{
SANDIPAN DASGUPTA ${ }^{1,3}$, SANJAY DEY², PAULOMI PAL ${ }^{3}$, BHASKAR MAZUMDER*3
}

1Department of Pharmacology, NSHM College of Pharmaceutical Technology, Kolkata, India, 700053, ${ }^{2}$ Assistant Professor, School of Pharmacy Techno India University, EM-4/2, Sector-V, Salt Lake, Kolkata, West Bengal, India, ${ }^{3}$ Department of Pharmaceutical Sciences, Dibrugarh University, Dibrugarh, Assam, India, 786004, India

Email: bhmaz@dibru.ac.in

Received: 22 Jul 2016 Revised and Accepted: 09 Sep 2016

\begin{abstract}
Objective: A simple, reliable, sensitive and validated reversed phase-high performance liquid chromatography (RP-HPLC) method was developed for quantification of lornoxicam (LX) in rat plasma.

Methods: Solid lipid nanoparticle (SLN) and nanostructured lipid carriers (NLC) gel formulations containing lornoxicam were prepared using highspeed homogenization followed by ultra-sonication. Pharmacokinetic study of formulated LX loaded SLN and NLC were performed on Wister albino rats.

Results: The chromatographic separation was performed on hypersil octadecylsilane (ODS)-18 column using a mobile phase of 10 mmol. Phosphate buffer $(\mathrm{pH}, 4.5)$ and acetonitrile $(65: 35 \mathrm{v} / \mathrm{v})$. Elute was monitored at $377 \mathrm{~nm}$ with a flow rate of $1 \mathrm{ml} / \mathrm{min}$. Calibration curve was linear over the concentration range of 25.38-2046.45 ng/ml. Retention times of LX and internal standard (piroxicam) were 9.3 and 10.2 min, respectively. Maximum plasma drug concentration, the area under the plasma drug concentration versus time curve and elimination half-life for LX loaded SLN gel were found $6381.51 \pm 971.27 \mathrm{ng} / \mathrm{ml}, 19917.21 \pm 7111.24 \mathrm{ng} \mathrm{h} / \mathrm{ml}$ and $7.27 \pm 1.21 \mathrm{~h}$ and $8558.13 \pm 1564.08 \mathrm{ng} / \mathrm{ml}, 21317.99 \pm 4568.71 \mathrm{ng} / \mathrm{ml}$ and $6.22 \pm 2.16 \mathrm{~h}$. respectively. In vivo in vitro correlation study, the fraction of drug dissolved from nanoparticle in $\mathrm{pH} 7.4$ was plotted against the fraction of drug absorbed and a linear correlation $\left(\mathrm{R}^{2}=0.9987\right)$ was obtained.
\end{abstract}

Conclusion: A novel simple, simple, sensitive, precise, rapid, accurate, and economical and reliable RP-HPLC method was developed and validated for the estimation of LX in rat plasma.

Keywords: Lornoxicam, SLN, NLC, RP-HPLC, Pharmacokinetic

(C) 2016 The Authors. Published by Innovare Academic Sciences Pvt Ltd. This is an open access article under the CC BY license (http://creativecommons.org/licenses/by/4. 0/) DOI: http://dx.doi.org/10.22159/ijpps.2016v8i11.14256

\section{INTRODUCTION}

Lornoxicam (LX), chemically 6-chloro-4-hydroxy-2-methyl- $\mathrm{N}$-2pyridinyl-2H-thienol [1, 2]-1,2-thiazine-3-carboxamide1,1-dioxide, is a member of the oxicam class of non-steroidal anti-inflammatory drugs (NSAIDs) with having analgesic, anti-inflammatory and antipyretic properties [1,2]. LX also shows a significant therapeutic effect in pain like osteoarthritis and rheumatoid arthritis [3, 4]. LX acts by non-selective inhibition of cyclooxygenase- 1 and-2. Quantification of the active component in a dosage form is essential to the quality control of these systems. Quality control checks the suitability of a drug delivery system for the intended application. It serves as a marker for the consistency and predictability of the performance of dosage forms. RP-HPLC methods are widely reported for the quantitative estimation of LX but to this day, no reports are available on the quantification of LX in nanostructured delivery systems such as solid lipid nanoparticles (SLN) and nanostructured lipid carriers (NLC) gel. Reversed phase high performance liquid chromatography (RP-HPLC) assembled with photodiode array (PDA) detector is most widely used in industries and research base organization for quantification of drugs present in the plasma sample. Several analytical methods for determination of LX in plasma and pharmaceutical formulation using UV spectroscopy $[5,6]$, RP-HPLC $[7,8]$ and polarography [9] have been reported. Several modern analytical techniques like liquid chromatographicelectrospray ionization tandem mass spectromicroscopy for the quantification of LX in the biological sample have been described in different research article [10]. However, the reported methods suffer from drawbacks such as poor resolution, cumbersome, complex sample preparation; require a sophisticated instrument, and more analytical expenses. Several RP-HPLC methods were reported in the literature for the quantitative determination of LX in biological samples. However, most of the published methods described the quantification of LX along with other drugs by RPHPLC technique. To best our knowledge, a validate RP-HPLC method for the determination of LX loaded SLN and NLC in a biological sample have not been reported.

Hence in this paper, we reported a simple, sensitive, precise, rapid, accurate, and economical, reliable, and validated RP-HPLC method requiring simple sample preparation and short running time for the determination of pharmacokinetic profile of LX after transdermal administration of SLN and NLC in rats.

\section{MATERIALS AND METHODS}

LX was obtained as gift sample from Akumbs Drugs, (Hridwar, India). Stearic acid, pluronicF68, carbopol 940 P, and oleic acid were purchased from Himedia, Mumbai, India. phospholipon90G (phosphatidylcholine 90\%) was collected as gift sample from $\mathrm{GmbH}$ Germany. HPLC grade acetonitrile, methanol, and dichloromethane were procured from E-Merck, Mumbai. Potassium dihydrogen phosphate $\left(\mathrm{KH}_{2} \mathrm{PO}_{4}\right)$ and orthophosphoric acid $\left(\mathrm{H}_{3} \mathrm{PO}_{4}\right)$ were purchased from SD Fine Chem. Ltd., Mumbai, India. All other chemicals and solvents used were of analytical grade. Water used in the HPLC analysis was prepared by the water purifier (AriumR, 611UF, Sartorius, Germany). The mobile phase and all the solutions were filtered through a $0.45 \mu \mathrm{m}$ ultipor ${ }^{\circledR} \mathrm{N} 66^{\circledR}$ membrane filter (Pall Life Sciences, USA) prior to use.

\section{Instruments}

HPLC system (Waters, USA) consisting of the quaternary pump (WaterTM600), 7725i rheodyne manual injector, PDA detector, and empower-II software and these assembled were used for analysis. They were processed by using of micropipettes (Ependruff, USA), spinix 
vortexer (M37610-33, Barnstead International, USA), biofuge fresco centrifuge (Heraeus, Germany), ultra-sonicator (LobaChem, Mumbai), nitrogen gas evaporator, and multi-pulse vortexed (Glas-COL, USA).

\section{Experimental animals}

Wister rats of either sex weighing 130-140 gm, housed at a temperature of $22 \pm 1{ }^{\circ} \mathrm{C}$ and relative humidity of $55 \pm 10 \%$ in controlled room were used in the experiments. The pharmacokinetic studies were conducted under protocol number MC/UCMS/IAEC/DU/12 of the Institutional Animal Ethical Committee of Department of Pharmaceutical Sciences, Dibrugarh University, Dibrugarh, Assam. The experiments were conducted in accordance with the guidelines provided by the Committee for the Purpose of Control and Supervision of Experiments on Animals (CPCSEA, Govt. of India).

\section{Preparation of Lx-loaded SLN and NLC}

SLN and NLC were prepared by high-speed homogenization and ultrasonication method [11]. Briefly, LX and phospholipon 90G were dissolved in methanol. Stearic acid was dissolved in acetone and mixed with methanol containing LX and phospholipon 90G. The mixture was added dropwise to an aqueous solution of pluronic F68 and maintained at $75^{\circ} \mathrm{C}\left(5^{\circ} \mathrm{C}\right.$ above the melting point of the lipid). A pre emulsion thus obtained was stirred using an ultra-turrax T25 (IKA-Werke GmBH, Germany) at $15000 \mathrm{rpm}$ for $3 \mathrm{~min}$. This preemulsion was immediately placed in a probe-type sonicator $(6 \mathrm{~mm}$ diameter, $20 \mathrm{~W}$; Malvern Instruments, UK) by an ultrasonic processor to prevent crystallization. After 15 min of ultrasonication, the nanodispersion was kept for cooling under continuous stirring. During this process, SLN was formed by recrystallization. The prepared SLN dispersions were lyophilized for further studies. NLC was prepared in a similar manner wherein only the "solid lipid" (Stearic acid) was replaced by oleic acid in different proportions [12]. The formulated nanoparticles were evaluated for drug entrapment efficiency, particle size measurement, X-Ray diffraction study, scanning electron microscopy and in vitro drug release study. The in vitro drug release study was performed in franz diffusion cell with the magnetic stirring rate at $800 \mathrm{rpm}$. The release medium was contained 25 $\mathrm{ml}$ of phosphate buffer ( $\mathrm{pH} 7.4$ ). At predetermined intervals $2 \mathrm{ml}$ of samples were withdrawn from receptor compartment and replaced with equal volume of release medium [11].

\section{Chromatographic conditions}

HPLC analysis was performed using a hyper sim ODS C18 column $(250 \mathrm{~mm} \times 4.6 \mathrm{~mm}$, particle size $5 \mu \mathrm{m})$. The mobile phase consisted of phosphate buffer $(\mathrm{pH}, 4.5)$ and acetonitrile in the ratio of 65:35 $\% \mathrm{v} / \mathrm{v}$. Eluent was monitored with the PDA detector at $377 \mathrm{~nm}$ with a flow rate of $1 \mathrm{ml} / \mathrm{min}$ and sample of $20 \mu \mathrm{l}$ was injected in the column and the temperature of the RP-HPLC system was maintained at a temperature of $30 \pm 2{ }^{\circ} \mathrm{C}$ all over the study.

\section{Preparation of standard solution}

A stock solution of LX $(50 \mu \mathrm{g} / \mathrm{ml})$ and piroxicam (PX) (internal standard, $50 \mu \mathrm{g} / \mathrm{ml}$ ) were prepared in acetonitrile. Further dilution of a stock solution of LX was carried out using mobile phase for the preparation of working standard solution. Calibration standards were prepared freshly by spiking working standard solution of LX into the blank plasma to get the final concentration of 25.38, 50.77, $101.53,203.06,406.12,846.09,1244.24,1637.16,2046.45 \mathrm{ng} / \mathrm{ml}$. All the solutions were prepared once and analyzed daily over a period of $3 \mathrm{~d}$ for the inter-day precision of the method. These solutions were stored at $-20^{\circ} \mathrm{C}$ until further study [13].

\section{Quality control standards}

Lowest quality control standards, median quality control standards, and highest quality control standards were prepared by spiking drugfree plasma with LX to give solutions containing 101.53, 406.12 and $1244.24 \mathrm{ng} / \mathrm{ml}$, respectively. They were stored at $-20 \mathrm{C}$ till analysis.

\section{Sample preparation}

An aliquot quantity of $100 \mu \mathrm{l}$ of rat plasma spiked with $10 \mu \mathrm{l}$ of calibration standard solution of LX was taken in a $2 \mathrm{ml}$ stopper centrifuge tube and mixed for $10 \mathrm{~s}$. To this, $10 \mu \mathrm{l}$ of internal standard (IS) solution $(50 \mu \mathrm{g} / \mathrm{ml})$ was added and mixed for $20 \mathrm{~s}$. The drug was extracted by vortexing with $1.5 \mathrm{ml}$ of selected extracting solvents (acetonitrile, methanol and di-chloromethane) in a spinix vortexer for $10 \mathrm{~min}$. followed by centrifugation at 10,000 rpm for $5 \mathrm{~min}$ at 4 ${ }^{\circ} \mathrm{C}$. The supernatant was withdrawn and dried under nitrogen at 25 psi for $10 \mathrm{~min}$ at $40^{\circ} \mathrm{C}$. The residue was reconstituted with $100 \mu \mathrm{l}$ of mobile phase and $20 \mu \mathrm{l}$ was injected onto the RP-HPLC column [13].

\section{Validation of method}

The validation of an analytical method confirms the characteristics of the method to satisfy the requirements of the application domain [13]. The method was validated according to ICH guidelines $[14,15]$ for specificity, recovery, linearity, precision and stability. Under the validation programme the following parameters were studied:

\section{Specificity}

The specificity criterion demonstrates that the result of the method is not affected by the presence of interferences, i.e. whether the compound of interest elutes without interfering with other compounds and components of plasma. The specificity of the method was determined by comparing the chromatograms obtained from the aqueous samples of LX and IS with those obtained from blank plasma. Blank plasma samples from each of five rats were processed in the presence of IS and another set of five samples processed with LX and IS to evaluate the presence of interference around the peak of LX. The median quality control solution $(406.12$ $\mathrm{ng} / \mathrm{ml}$ ) of LX was injected into the column under the optimized chromatographic conditions to obtain the chromatographic peaks of LX and I. S. so as to differentiate them from the interfering peaks of plasma components.

\section{Selection of solvent for recovery of drug}

The recovery of an analyte is the extraction efficiency of an analytical process, reported as a percentage of the known amount of an analyte carried through the sample extraction and processing steps of the method. Different organic extraction solvents (acetonitrile, methanol, ethanol, hexane, dichloro methane and chloroform) were tried in the experiment to recover LX from plasma samples. Quality control samples were prepared in triplicate at three levels of $101.53,406.12$ and $1244.24 \mathrm{ng} / \mathrm{ml}$ of LX and assayed by HPLC method as described as above. The extraction efficiency of LX was determined by comparing the peak areas obtained from extracted quality control samples with the peak area of an aqueous working solution containing the same concentration of LX at three levels.

\section{Linearity}

Quantitative analytical results are highly influenced by the quality of the calibration curve $[16,17]$. Nine different concentrations of LX with a fixed concentration of IS in blank plasma were processed, and a calibration curve was constructed in the specified concentration range $(25.38,50.77,101.53,203.06,406.12,846.09,1244.24$, 1637.16 , and $2046.45 \mathrm{ng} / \mathrm{ml}$ ). The calibration curve was plotted between the ratio of peak areas of LX to IS and concentration of LX by replicate analysis $(n=6)$ at all concentration levels and the linear relationship was evaluated using the least square method using Microsoft excel ${ }^{\circledR}($ Microsoft Corporation, USA) program.

\section{Precision and accuracy}

Both repeatability (within a day precision) and reproducibility (between days precision) were determined as follows. Three quality control samples (lowest, median, and highest) were subjected for the study. Each of the specified quality control samples was injected five times for determination of repeatability (five different time intervals) and repeatability (over a period of $5 \mathrm{~d}$ ). Mean and relative standard deviation were calculated and used to predict the accuracy and precision of the method.

\section{Stability studies}

The quality control standards containing 101.53, 406.12, and $1244.24 \mathrm{ng} / \mathrm{ml}(\mathrm{n}=6)$ of $\mathrm{LX}$ were subjected for detection of stability of LX in rat plasma. The initial assay of the samples was conducted. The quality control samples were divided into three set each set 
contained five samples. The first set was kept in polypropylene tubes and subjected to three freezes $\left(-20^{\circ} \mathrm{C}\right.$ for $24 \mathrm{~h}$ )-thaw (room temperature for $24 \mathrm{~h}$ ) cycles. The second set was kept at room temperature for $24 \mathrm{~h}$ and the third set was kept at room temperature for $1 \mathrm{mo}$. All the samples were analyzed by standard chromatographic conditions to determine their peak areas. Samples were considered to be stable when the final assay values of samples were found similar to that of the initial assay value of the drug.

\section{Pharmacokinetic study in rats}

The method described above was applied to quantify the plasma concentration of LX in a single-dose pharmacokinetic study conducted on six Wister rats. The protocol was approved by the Institutional Ethical Committee at the Dibrugarh University, Dibrugarh, India. The experiments were conducted as per CPCSEA (Committee for Prevention, Control and Supervision of Experimental Animals) guidelines. The rats weighing 130-150 gm were housed with free access to food and water, except for the final $12 \mathrm{~h}$ before experimentation. After transdermal application of formulated nanoparticle containing $2.5 \mathrm{mg}$ of $\mathrm{LX}, 2 \mathrm{ml}$ of blood samples were collected from the tail vein at $0,0.25,0.5,1,2,3,4,6,9,12$ and $24 \mathrm{~h}$ time points into heparinized collection tubes. The blood was immediately centrifuged $(1000 \times \mathrm{g})$ for $10 \mathrm{~min}$ at an ambient temperature. The supernatant plasma layer was separated and stored at $-20^{\circ} \mathrm{C}$ until analyzed. The plasma samples were analyzed for LX concentrations by validated RP-HPLC method. The first order elimination rate constant $\left(\mathrm{k}_{\mathrm{el}}\right)$ was estimated by the least square regression of plasma concentration-time data points of the curves describing the terminal log-linear decaying phase. The plasma halflife $\left(\mathrm{t}_{1 / 2}\right)$ was calculated from $\mathrm{k}_{\mathrm{el}}\left(\mathrm{t}_{1 / 2}=0.693 / \mathrm{k}_{\mathrm{e} 1}\right)$. The area under the plasma concentration-time curve from zero to the last measurable plasma concentration at time $\mathrm{t}\left(\mathrm{AUC}_{0-\mathrm{t}}\right)$ was calculated by applying the trapezoidal rule. The area was extrapolated to infinity $\left(\mathrm{AUC}_{0-\infty}\right)$ by the addition of $\mathrm{Ct} / \mathrm{k}_{\mathrm{el}}$ to $\mathrm{AUC}_{0-\mathrm{t}}$, where $\mathrm{C}_{\mathrm{t}}$ is the last detectable drug concentration. The absorption rate constant $\left(\mathrm{k}_{\mathrm{a}}\right)$ was determined by the residual method. The maximum observed $\mathrm{LX}$ concentration $\left(\mathrm{C}_{\max }\right)$ and the time at which $\mathrm{C}_{\max }$ was observed $\left(\mathrm{T}_{\max }\right.$ ) were obtained directly from the profile. The AUMC AUC ${ }_{0-\infty}$ is the area under the plot of time vs. the product of time and concentration extrapolated to infinity and was calculated by trapezoidal rule. The mean residence time (MRT) was determined by AUMC divided by AUC. All the pharmacokinetic parameters were calculated using microsoft ${ }^{\circledR}$ office excel 2003 (Microsoft Corporation, USA) software application. [12]

The Wagner-Nelson method [18] was applied to deconvolute the percentage of the LX absorbed using Eq. (1).

$$
\mathrm{F}_{\mathrm{t}}=\mathrm{C}\left[\mathrm{C}_{\mathrm{t}}+\mathrm{Ke}_{\mathrm{e}} \mathrm{AUC} \mathrm{C}_{(0-\infty)}\right] \ldots \ldots \ldots \ldots \ldots \ldots . .(1)
$$

Where, $F_{t}$ is the amount of drug absorbed. The fraction absorbed (FRA) was calculated using eq. (2) and was plotted against the fraction of drug dissolved (FRD) at the same time and the linear regression analysis was used to examine the in vitro-in vivo relationship.

$$
\text { Fraction absorbed(FRA) } \left.=\mathrm{C}_{\mathrm{t}}+\mathrm{K}_{\mathrm{el}} \mathrm{AUC}_{(0-\mathrm{t})} / \mathrm{K}_{\mathrm{el}} \mathrm{AUC}_{(0-\infty)}\right] \ldots \ldots . . .(2)
$$

\section{Establishment of in vivo in vitro correlation (IVIVC)}

An IVIVC refers to the predictive mathematical model describing the relationship between in vitro property of a controlled-release dosage form (usually the rate or extent of drug dissolution or release) and a relevant in vivo response, such as plasma drug concentration or amount of drug absorbed. A level A correlation is usually estimated by a two-stage procedure: deconvolution followed by comparison of the fraction of drug absorbed (FRA) to the fraction of drug dissolved (FRD). A correlation of this type represents a point-to-point relationship between in vitro dissolution and the in vivo input rate [19]. Accordingly, the deconvolution procedure was done on nanoparticles using mean LX plasma concentration vs. time profile using the method of wagner-nelson. The data of FRA (obtained by deconvolution) and FRD for each formulation were then plotted to develop an IVIVC model. The linear, quadratic, and cubic models (Eq. (2) bearing the following forms were tested to obtain the best fit:

$$
Y=a+b X, Y=a+b X+c X^{2}, Y=a+b X+c X^{2}+d X^{3}
$$

where $a, b, c, d$ represent regression parameters associated with each function, $\mathrm{Y}$ is the FRA in vivo and $\mathrm{X}$ is the FRD in vitro. The coefficients of determination $\left(\mathrm{r}^{2}\right)$ for all developed models were determined. The highest $\mathrm{r}^{2}$ value equation was considered as the best fitting model.

\section{Statistical analysis}

The linearity of the proposed method was evaluated by measuring the single factor analysis of variance (ANOVA) of the linear regression data. Statistical significance was established by a $P$ value $<0.05$, which indicates that the model is explained by the proposed regression at a $95 \%$ confidence interval.

\section{RESULTS AND DISCUSSION}

\section{Preparation of SLN and NLC}

Both SLN and NLC formulations were successfully prepared using high-speed homogenization method. The particle size, zeta potential, and poly dispersity index were found to be within the range of 140$193 \mathrm{~nm} \pm 0.220,-22$ to $-32 \mathrm{mV}$, and $0.354-0.301$ for SLN formulations and $146-201 \mathrm{~nm} \pm 0.232,-23$ to $-30 \mathrm{mV}$, and $0.355-$ $0.354 \pm 0.145$ for NLC formulations, respectively. The entrapment efficacy of the SLN formulations was found within the range of $42 \%$ $72 \%$ and that for the NLC formulations was $61 \%-79 \%$. Thermal analyses such as DSC, XRD showed good results. In vitro permeation rate and controlled release property of carbopol-loaded SLN, NLC gel was studied.

\section{Selection and development of chromatographic method}

Normal phase chromatography can be used for the separation of non-ionic and non-polar substances, while reversed-phase chromatography ( $\mathrm{C}_{8}$ and $\mathrm{C}_{18}$ column) can be used for the separation of non-ionic as well as ionic non-polar to semi-polar substances. Thus, LX (an ionizable semi-polar weak acid) can satisfactorily separated by reversed phase chromatography. However, octylsilane columns are less retentive as compared to octadecylsilane. The majority of the ionizable pharmaceutical compounds can be very well separated in octadecylsilane reversed phase columns [16]. Therefore, octadecylsilane column was considered for isolation of LX from the plasma sample. A mixture of methanol and phosphate buffer $(\mathrm{pH} 4.5)$ was used as a mobile phase for the analysis of $\mathrm{LX}$ [14]. The optimum ratio of acetonitrile to phosphate buffer $(\mathrm{pH} 4.5)$ used in the current investigation was $65: 35 \% \mathrm{v} / \mathrm{v}$, which was selected on the basis of best resolution and absence of tailing and fronting. The best resolution and sensitivity of the peaks of LX and IS were obtained at $377 \mathrm{~nm}$ and flow rate of $1 \mathrm{ml} / \mathrm{min}$ of the mobile phase.

\section{Validation of methods}

\section{Specificity}

Typical chromatogram (fig. 1) of a mixture of LX and IS revealed that they are well separated under the RP-HPLC conditions applied. A chromatogram of blank plasma sample is shown in fig. II. Retention time was found $9.3 \mathrm{~min}$ for LX and $10.4 \mathrm{~min}$ for IS. In the comparison of a chromatogram of LX and IS (fig. 1) with the chromatogram of blank plasma (fig. 2), the absence of interference of plasma components is observed around the zone of the retention time of LX and IS. The chromatogram of the medium level quality control sample (i.e. $406.12 \mathrm{ng} / \mathrm{ml}$ ) of LX spiked with rat plasma (fig. 3) showed a good resolution peaks for LX and IS which are well differentiated from the peaks of plasma components.

\section{Selection of extracting solvent for recovery of drug}

On the basis of capability of solvent to extract the maximum amount of drug from plasma was selected for extracting of LX from rat plasma. The recovery efficiency of $\mathrm{LX}$ from rat plasma was found to be $83.52 \pm 0.78$, $26.26 \pm 1.46,59.04 \pm 1.68,76.46 \pm 0.89,62.88 \pm 1.05$, and $71.08 \pm 1.25 \%$ in acetonitrile, ethanol, methanol, hexane, dichloromethane and chloroform, respectively. Among the different solvents, acetonitrile showed maximum recovery efficiency, and therefore, acetonitrile was selected as the extracting solvent for extraction of LX from rat plasma. 


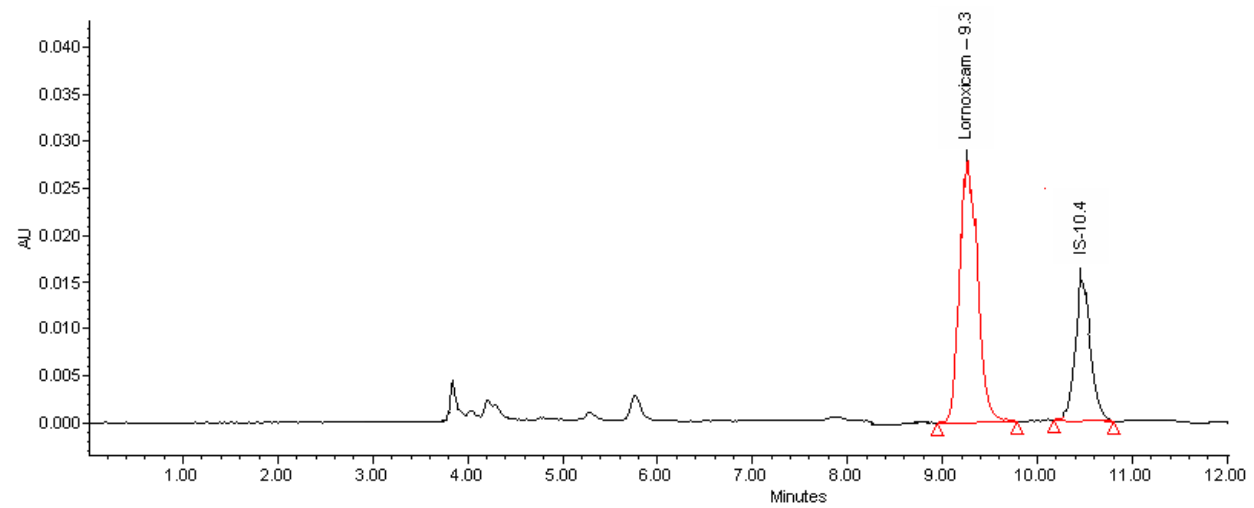

Fig. 1: Chromatogram of the mixture of $\mathrm{LX}$ and internal standard in mobile phase

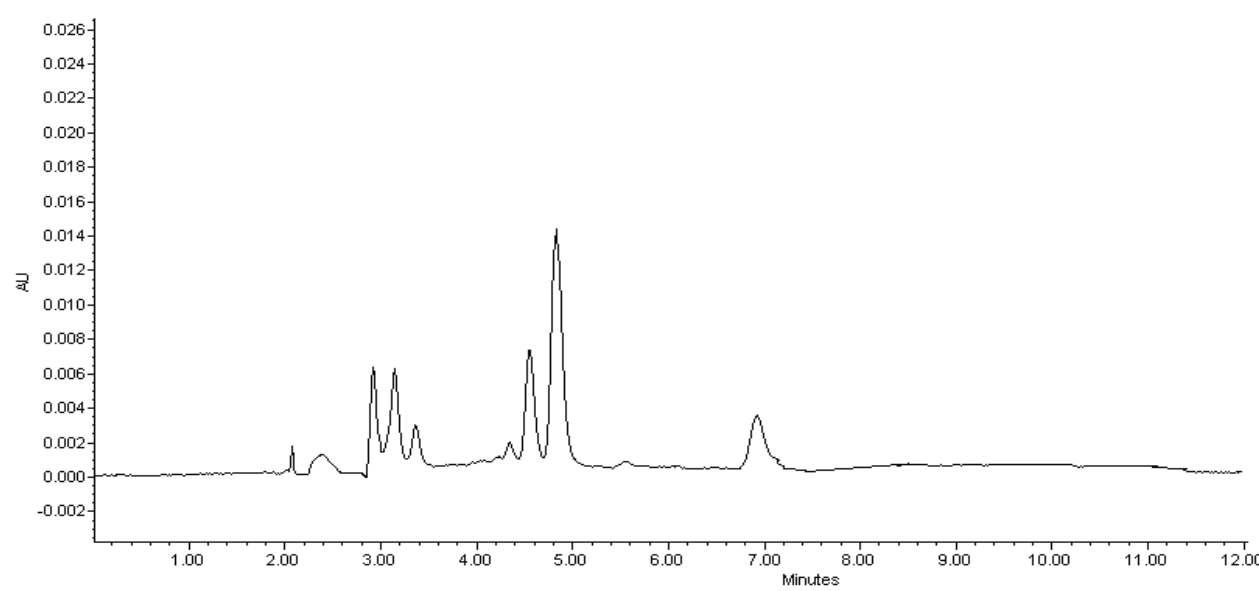

Fig. 2: Chromatogram of blank plasma sample

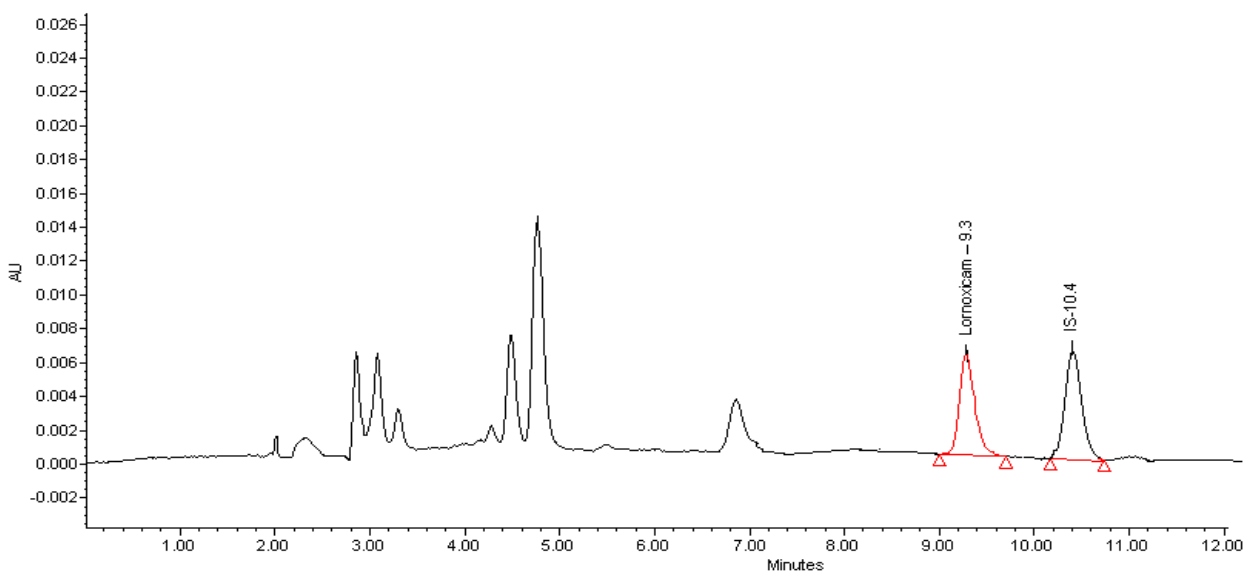

Fig. 3: Chromatogram of drug along with IS in plasma sample

\section{Linearity}

The ratio of peaks area of LX to IS at various concentrations of LX in plasma was determined. The chromatographic responses (ratio of peaks area of LX to IS vs. LX concentration) were found to be linear over an analytical range of $25.38-2046.45 \mathrm{ng} / \mathrm{ml}$ with regression coefficient $\left(\mathrm{r}^{2}\right)$ value 0.999 which showed good linearity. The linear regression equation obtained was $\mathrm{Y}=0.001 \mathrm{X}+0.013$.

\section{Precision and accuracy}

The accuracy of the proposed RP-HPLC method was determined by using three quality control samples, and the results are reported in table 1. The relative standard deviation (RSD) of the intra-day assay of the drug was ranged from 3.51 to $5.35 \%$ and for the inter-day assay was from 2.77 to $3.16 \%$ (table 2).

\section{Stability}

The result of the stability of LX in rat plasma at different conditions is presented in table 3 . The result revealed that the final drug concentration of each quality control sample at three freeze-thaw cycles and the sample stored at room temperature for $24 \mathrm{~h}$ and $1 \mathrm{mo}$ were almost similar with the initial concentration. The RSD value ( = 6) of final concentration of LX after three freeze-thaw cycles, $24 \mathrm{~h}$ and 1 mo was $<10.87,<9.69$ and $<7.01$, respectively. 
Table 1: Peak area of the chromatogram of lornoxicam and I. S. at different concentration of lornoxicam

\begin{tabular}{llll}
\hline Concentration of $\mathbf{L X}(\mathbf{n g} / \mathbf{m l})$ & \multicolumn{2}{l}{ Peak area of the chromatogram } & \multicolumn{1}{c}{ Ratio of peak area } \\
\cline { 2 - 3 } & Lornoxicam & I. S. (Piroxicam) & 0.0336 \\
25.38 & 10590 & 315383 & 0.0595 \\
50.77 & 19414 & 326485 & 0.1477 \\
101.53 & 48744 & 296772 & 0.3064 \\
203.06 & 90937 & 298161 & 0.6365 \\
406.12 & 189793 & 340716 & 1.2257 \\
846.09 & 417630 & 333671 & 1.7397 \\
1244.24 & 580504 & 333077 & 2.3998 \\
1637.16 & 799309 & 335589 & 2.8629 \\
2046.45 & 960772 & & \\
\hline
\end{tabular}

LX: Lornoxicam IS: internal standard, ng/ml: nanogram per milliliter

Table 2: Intra-and inter-day precision and accuracy for lornoxicam

\begin{tabular}{|c|c|c|c|c|c|c|}
\hline \multirow[t]{2}{*}{ QC sample (ng/ml) } & \multicolumn{3}{|c|}{ Intra-day variation } & \multicolumn{3}{|c|}{ Inter-day variation } \\
\hline & Observed value* & R. SD\% & Accuracy \% & Observed value* & R. SD\% & Accuracy \% \\
\hline 101.53 & $96.42 \pm 5.16$ & 5.35 & 94.97 & $103.14 \pm 3.26$ & 3.16 & 101.59 \\
\hline 406.12 & $428.31 \pm 12.63$ & 2.95 & 105.46 & $395.49 \pm 10.72$ & 2.71 & 97.38 \\
\hline 1244.24 & $1115.18 \pm 39.17$ & 3.51 & 89.63 & $1106.47 \pm 30.69$ & 2.77 & 88.93 \\
\hline
\end{tabular}

(mean \pm SD; $n=6), Q C$ : Quality control, RSD: relative standard deviation ng/ml: nanogram per mililiter

Table 3: Stability of LX in rabbit plasma

\begin{tabular}{|c|c|c|c|c|}
\hline Stability & QC sample (ng/ml) & Observed value* & R. SD\% & Accuracy \% \\
\hline \multirow[t]{3}{*}{ Freeze-thaw stability } & 101.53 & $105.23 \pm 9.38$ & 8.91 & 103.64 \\
\hline & 406.12 & $384.75 \pm 41.81$ & 10.87 & 94.74 \\
\hline & 1244.24 & $1121.47 \pm 68.58$ & 6.12 & 90.13 \\
\hline \multirow[t]{3}{*}{ Short term $(24 \mathrm{~h})$} & 101.53 & $96.98 \pm 6.69$ & 6.90 & 95.52 \\
\hline & 406.12 & $374.09 \pm 36.24$ & 9.69 & 92.11 \\
\hline & 1244.24 & $1167.57 \pm 81.14$ & 6.95 & 93.84 \\
\hline \multirow[t]{3}{*}{ Long term (30 d) } & 101.53 & $109.08 \pm 7.14$ & 6.55 & 107.44 \\
\hline & 406.12 & $384.57 \pm 26.94$ & 7.01 & 94.69 \\
\hline & 1244.24 & $1178.41 \pm 101.65$ & 8.63 & 94.71 \\
\hline
\end{tabular}

$($ mean $\pm S D ; n=6) . n g / m l:$ nanogram per mililiter RSD: relative standard deviation LX: Lornoxicam

\section{Pharmacokinetic study in rats}

The validated RP-HPLC method was applied to carry out a pharmacokinetic study of LX in rats. The mean plasma concentration of LX versus time profile following a single transdermal application of prepared LX loaded SLN and NLC on six rats is presented in fig. 4. The various pharmacokinetic parameters are summarized in table 4 .

\section{In vitro-in vivo correlation (IVIVC)}

To assess the viability and validity of the sustaining nature of nanoparticles, IVIVC study is essential, since prolonged release products may be especially suited for this kind of study. When the FRD from nanoparticles in $\mathrm{pH} 7.4$ was plotted against the FRA, a linear correlation was obtained (fig. 5).

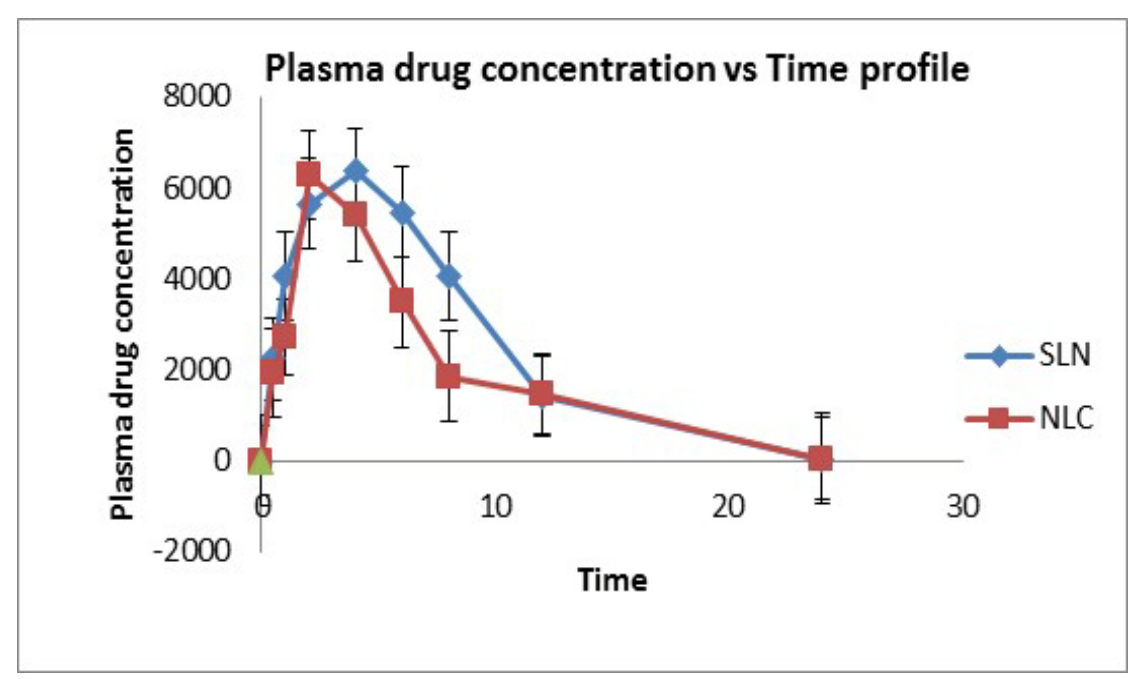

Fig 4: Mean plasma concentration versus time profile of loraxicam following transdermal delivery of lipid nanocarrier to rats $(\mathrm{n}=6)$. 
Table 4: Pharmacokinetic parameters of Lornoxicam as lipid nanocarrier to healthy rabbit volunteers

\begin{tabular}{|c|c|c|}
\hline \multirow[t]{2}{*}{ Pharmacokinetic parameter } & \multicolumn{2}{|l|}{ Observed value } \\
\hline & SLN & NLC \\
\hline Maximum plasma concentration, $C_{\max }(\mathrm{ng} / \mathrm{ml})$ & $6381.51 \pm 971.27$ & $8558.13 \pm 1564.08$ \\
\hline Time required to reach maximum plasma concentration, $T_{\max }(\mathrm{h})$ & $4.00 \pm 0.00$ & $2.00 \pm 0.00$ \\
\hline Area under the curve, $\mathrm{AUC}_{0-\infty}(\mathrm{ng} \mathrm{h} / \mathrm{ml})$ & $19917.21 \pm 7111.24$ & $21317.99 \pm 4568.71$ \\
\hline Area under momentum curve, AUMC $_{0-\infty}\left(\mathrm{ng} \mathrm{h}^{2} / \mathrm{ml}\right)$ & $122052.26 \pm 25847.08$ & $124310.20 \pm 19989.26$ \\
\hline Elimination half-life, $t_{1 / 2}(\mathrm{~h})$ & $7.27 \pm 1.21$ & $6.22 \pm 2.16$ \\
\hline Elimination rate constant, $K_{\mathrm{el}}\left(\mathrm{h}^{-1}\right)$ & $0.095 \pm 0.101$ & $0.111 \pm 0.086$ \\
\hline Mean residence time, MRT (h) & $6.128 \pm 1.057$ & $5.831 \pm 1.213$ \\
\hline
\end{tabular}

$($ mean $\pm S D ; n=6)$. SLN: solid lipid nanoparticle formulation, NLC: nanostructured lipid carrier formulation.

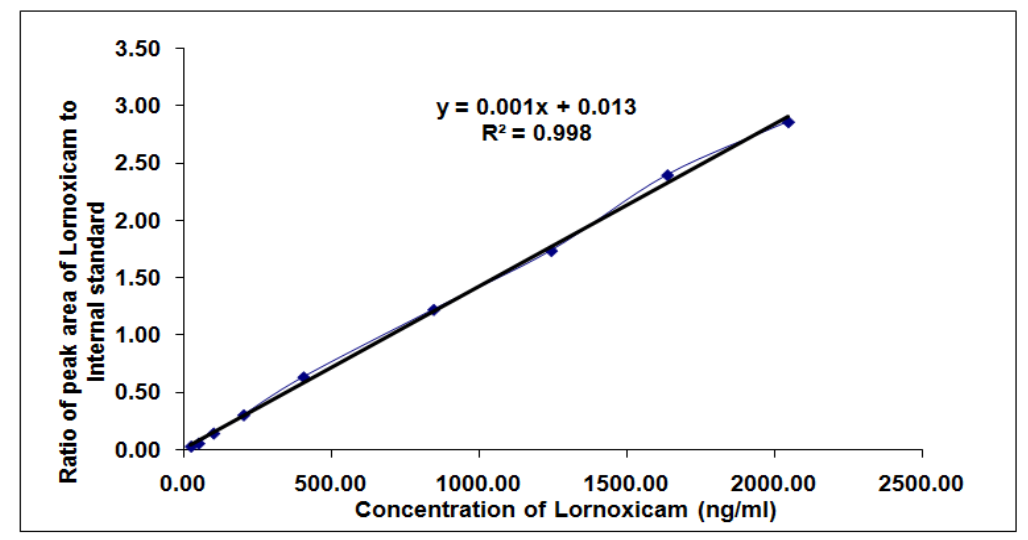

Fig. 5: Standard curve for In vitro \& In vivo co-relation between LX \& Internal standard VS. Conc. of LX

\section{DISCUSSION}

To develop a suitable and robust RP-HPLC method for the determination of lornoxicam from nanoparticles different mobile phases and columns were employed to achieve the efficient separation and resolution. The result shows that within the concentration range mentioned above, there was an excellent correlation between peak area and concentration of drug [20]. The chromatogram of medium level quality control sample (i.e. 406.12 $\mathrm{ng} / \mathrm{ml}$ ) of LX spiked with rat plasma (fig. 3) showed a good resolution peaks for LX and IS which are well differentiated from the peaks of plasma components, which shows that the analyte peaks were pure, and there were no interferences from formulation excipients in the analyte peak. Accuracy data ranged from $94.97 \%$ to $89.63 \%$ for both the conditions indicated that there was no interference from endogenous plasma components [21]. Inter-day, as well as intra-day replicates of LX, resulted an RSD value less than 5.35 $\%$, which revealed that the high degree of precision of the proposed method. The accuracy of stored samples was found to be equivalent to $100 \%$. Hence, it can be inferred that LX was stable in rat plasma up to one month at room temperature. In the IVIVC study, a higher value of correlation coefficient suggested that a good correlation existed between in vitro-in vivo data. Finally a novel simple, simple, sensitive, precise, rapid, accurate, and economical and reliable RP-HPLC method was developed and validated for the estimation of LX in rat plasma using PDA detector. A maximum recovery of drug from plasma resulted by using acetonitrile as extracting solvent in comparison to other solvents. The results obtained proved that the method was accurate and reproducible, and drug was stable in rat plasma

\section{CONCLUSION}

Therefore, it can be concluded that the validated chromatographic condition is suitable for determination of pharmacokinetic profile of LX in rat plasma after transdermal application of SLN and NLC on rat and is also applicable to the routine therapeutic monitoring of the LX. A linear relationship was observed between FRD and FRA in IVIVC study.

\section{ACKNOWLEDGEMENT}

We acknowledge the financial support provided by University Grants Commission (UGC). We also acknowledge Akumbs Drugs,
Hridwar, India for supplying Lornoxicam. We also acknowledge Lipoid, GmbH Germany for supplying phospholipon 90G.

\section{CONFLICTS OF INTERESTS}

Declared none

\section{REFERENCES}

1. Bhatt PA, Hamrapurkar PD, Shigwan HS. Development and validation of stability indicating RP-HPLC method for Lornoxicam in bulk drug. Indian J Res Pharm Biotechnol 2013;4:959-66.

2. Singh B, Saini G, Sharma DNN, Roy SD, Gautam N. Antioxidant activities of two medicinal vegetables: Parkiajavanica and Phlogacanthusthyrsiflorus. Int J Pharm Sci Res 2011;2:102-6.

3. Hite GH, Foye WO. Analgesic. In: principles of medicinal chemistry. 5th Ed. Boston, Lippincott Wiliams and Wilkins; 2001. p. 771.

4. Müller RH, Lucks JS. Medication vehicles made of solid lipid particles (solid lipid nanospheres-SLN). Eur Patent; 1996.

5. Kim YH, H Ji, E Y, Park S, Chae S, Lee HS. Liquid chromatography-electrospray lonization tandem mass spectrometric determination of lornoxicam in human plasma. Arch Pharm Res 2007;30:905-10.

6. Patil KR, Shinde DB, Rane VP, SangshettiJr JN. Stabilityindicating LC method for analysis of lornoxicam in the dosage form. Chromatographia 2009;69:1001-5.

7. Yadav S, Jagtap AS, Rao JR. Simultaneous determination of paracetamol, Lornoxicam and Chlorzoxazone in tablets by highperformance thin layer chromatography. Pharm Chem 2012;4:1798-802.

8. Sivasubramanian L, Lakshmi KS, Tintu T. Simultaneous spectrophotometric estimation of Paracetamol and Lornoxicam in tablet dosage form. Int J Pharm Sci 2010;2:166-8.

9. Kim YH, Ji HY, Park ES, Chae SW, Lee HS. Liquid chromatography-electrospray ionization tandem mass spectrometric determination of Lornoxicam in Human Plasma. Arch Pharm Res 2007;30:905-10.

10. Dasgupta S, Ghosh SK, Ray S, Mazumder B. Solid lipid nanoparticles (SLNs) gels for topical delivery of lornoxicam in vitro and in vivo evaluation. Curr Drug Delivery 2013;10:656-66. 
11. Han F, Yin R, Che X, Yuan J, Cui Y, Yin H, et al. Nanostructured lipid carriers (NLC) based topical gel of flurbiprofen: design, characterization and in vivo evaluation. Int J Pharm 2012;439:349-57.

12. Pani NR, Acharya S, Patra S. Development and validation of RPHPLC method for quantification of glipizide in biological macromolecules. Int J Biol Macromol 2014;65:65-71.

13. Pani NR, Nath LK, Singh AV, Mahapatra SK. Development and validation of analytical method for the estimation of nateglinide in rat plasma. J Pharm Anal 2012;2:492-8.

14. Singh AV, Nath LK, Pani NR. Development and validation of analytical method for the estimation of lamivudine in rat plasma. J Pharm Anal 2011;1:251-7.

15. Bhattacharya SS, Banerjeeb S, Ghosh AK, Chattopadhyay P, Verma A, Ghosh A. An RP-HPLC method for quantification of diclofenac sodium released from biological macromolecules. Int J Biol Macromol 2013;58:354-9.

16. Hubert PH, Chiap P, Crommen J. The SFSTP guide on the chromatographic for drug bioanalysis: from the Washington conference to the laboratory. Anal Chim Acta 1999;391:135-48.
17. Bourne DWA. In: Banker GS, Rhodes CD. Eds. Modern Pharmaceutics (Drugs and the Pharmaceutical Sciences). 4th Vol. 121. Marcel Dekker Inc, NewYork; 2002. p. 67-92.

18. Wagner JG. Biopharmaceutics and Relevant Pharmacokinetics EDN first. Drug Intelligence Publishers, Illinois; 1971.

19. US Department of Health and Human Services, Guidance for the Industry, USFDA, CDER. Rockville; 2000.

20. Aher KB, Bhavar GB, Joshi HP. Rapid RP-HPLC method for quantitative determination of Lornoxicam in bulk and pharmaceutical formulations. Int $\mathrm{J}$ ChemTech Res 2011;3:1220-4

21. Ravisankar P, Devala GR. Development and validation of RPHPLC method for determination of levamisole in bulk and dosage form. Asian J Pharm Clin Res 2013;6:169-73.

\section{How to cite this article}

- Sandipan Dasgupta, Sanjay Dey, Paulomi Pal, Bhaskar Mazumder. RP-HPLC Method development, validation and quantification of lornoxicam in lipid nanoparticle formulations. Int J Pharm Pharm Sci 2016;8(11):152-158. 